\title{
Over 500 liver transplants including more than 400 living-donor liver transplants in 2019 at Asan Medical Center
}

\author{
Youngin Yoon, Sung-Gyu Lee, Deok-Bog Moon
}

Division of Hepatobiliary, Department of Surgery, Asan Medical Center, University of Ulsan College of Medicine, Seoul, Korea

Background: More than 400 liver transplants were performed at Asan Medical Center (AMC) in 2011, and over 500 liver transplants including 420 living-donor liver transplants (LDLTs) were performed in 2019. Herein, we report the methodology of these procedures.

Methods: Since the first adult LDLTs at AMC using the left and right lobes were successfully performed, various innovative techniques and approaches have been developed: modified right lobe, dual graft, donor exchange for $A B O$ incompatibility, expansion of indications and no-touch techniques for hepatocellular carcinoma, intraoperative cine-portogram and additional intervention for large collaterals, management of portal vein thrombosis (PVT) and stenosis, salvage LDLT after major hepatectomy, and timely LDLT for patients with acute-on-chronic liver failure.

Results: Four hundred twenty LDLTs in 403 adult and 17 pediatric patients and 85 deceased-donor liver transplants in 74 adult and 11 pediatric patients were performed. The number of deceased-donor liver transplants remained constant since 2011, but the number of LDLTs increased steadily. One hundred thirty patients $(25.7 \%)$ required urgent liver transplantations and 24 patients with acute-on-chronic liver failure underwent LDLT. PVT including grade 1, 2, 3, and 4 was reported in 91 patients (18.0\%), and Yerdel's grade 2, 3, and 4 PVT was reported in 47 patients (51.6\%); all patients with PVT were successfully treated. Adult LDLTs for hepatocellular carcinoma and ABO incompatibility accounted for $52.6 \%$ and $24.3 \%$ of the cases, respectively. In-hospital mortality in 2019 was $2.97 \%$.

Conclusions: Continual efforts to overcome challenging problems in LDLT with various innovations and dedication of the team members during the perioperative period to improve patient outcomes were crucial in increasing the number of liver transplantations at Asan Medical Center.

Corresponding author: Deok-Bog Moon

E-mail: mdb1@amc.seoul.kr

\section{(c) The Korean Society for Transplantation}

This is an Open Access article distributed under the terms of the Creative Commons Attribution Non-Commercial License (http://creativecommons.org/licenses/by-nc/4.0/) which permits unrestricted non-commercial use, distribution, and reproduction in any medium, provided the original work is properly cited. 